Supporting Information

\title{
Investigating Single-Molecule Fluorescence Spectral Heterogeneity of Rhodamines Using High-Throughput Single-Molecule Spectroscopy
}

\author{
Yang Zhang, ${ }^{*}+, £$ Yu Zhang, ${ }^{\ddagger}, £$ Ki-Hee Song, ${ }^{\dagger}$ Wei Lin, ${ }^{\ddagger}$ Cheng Sun, ${ }^{\S}$ \\ George C. Schatz, ${ }^{\ddagger}$ Hao F. Zhang ${ }^{\dagger}$ \\ ${ }^{\dagger}$ Departments of Biomedical Engineering, ${ }^{\S}$ Department of Mechanical Engineering, and \\ ${ }^{\ddagger}$ Department of Chemistry, Northwestern University, Evanston, IL60208, United States \\ *E-Mail: yang.zhangfl@gmail.com \\ $£$ Contributed equally
}

- Experimental Procedures.

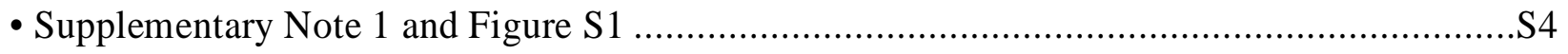

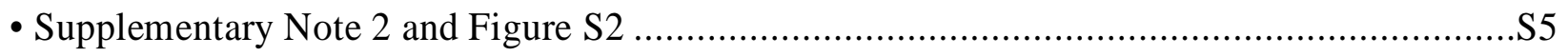

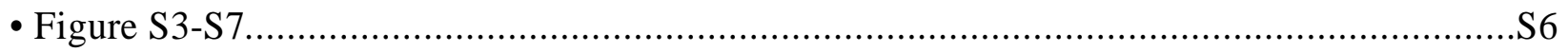

- Supplementary Note 3, Figure S8 and Computational Details .........................................S8

$\dagger$ Department of Biomedical Engineering, Northwestern University

$\ddagger$ Department of Chemistry, Northwestern University

$\S$ Department of Mechanical Engineering, Northwestern University 


\section{Experimental Procedures}

Materials and Methods. RhB, Rh101, Rh110, NR, AO, Pentacene, and Polystyrene (PS) were purchased from Sigma Aldrich. Perylene Diimide (PDI) is a gift from Prof. Michael Wasielewski's group at Northwestern Chemistry. Goat anti-mouse IgG-AF647 and IgG-TRITC conjugates were purchased from ThermoFisher. Single-molecule samples except for AF647-IgG and TRITC-IgG conjugates were directly deposited or doped with PS and then deposited on glass substrates with a Laurell WS-650SZ-23NPPB spin coater at 2,000 rpm for $60 \mathrm{~s}$ on No.1 cover glasses (22 mm× $22 \mathrm{~mm}$ ). Prior to spin coating cover glasses were cleaned with a South Bay Technology PC 2000 Plasma Cleaner. The emission spectrum of Pentacene in Chloroform solution (saturated, $\sim 1 \mu \mathrm{M}$ ) is measured using a Shimadzu Spectrofluorometer RF-6000 (excitation wavelength is $532 \mathrm{~nm}$ ).

\section{Preparation of single-molecule samples.}

For AF647-IgG and TRITC-IgG single molecules deposited on glass, $2 \mu \mathrm{g} \mathrm{mL} \mathrm{mL}^{-1}$ of AF647-IgG or TRITC-IgG conjugates in PBS $(200 \mu \mathrm{L})$ was added to plasma-cleaned cover glass for 5 min. The glass was then rinsed thoroughly with PBS and imaged with an imaging buffer.

For single molecules deposited directly on glass, solutions of RhB, AO, Rh101, Rh110, PDI, NR, and PDI (10 nM, EtOH, $100 \mu \mathrm{L}$ ) were added to plasma-cleaned cover glass, spin-coated at $2000 \mathrm{rpm}$ for $60 \mathrm{~s}$, and dried under reduced pressure overnight.

For single molecules doped in Polystyrene-coated glass film, solutions of RhB or NR (10 nM, EtOH) were mixed with Polystyrene $\left(\mathrm{Mn}_{\mathrm{n}}=300 \mathrm{kDa}, 10 \mathrm{mg} \mathrm{mL}^{-1}\right.$, Toluene) and then added to plasma cleaned cover glass, spin-coated at 2000 rpm for $60 \mathrm{~s}$ and dried under reduced pressure overnight.

Optical setup. The detailed setup of our sSMLM system is reported previously ${ }^{2}$ and illustrated in Figure 1. Briefly, the excitation sources were $488 \mathrm{~nm}$ (Rh110), 532 nm (RhB, Rh101, PDI, Pentacene, and NR), and 642 nm (AF647) continuous-wave laser with maximal powers of $100 \mathrm{~mW}, 200 \mathrm{~mW}$, and $100 \mathrm{~mW}$ respectively. The power densities of the laser output measured at the back port of the microscope are $\sim 50-200 \mathrm{~W} \mathrm{~cm}^{-2}$. The samples were mounted on the microscope stage and imaged under a Total Internal Reflection Fluorescence (TIRF) objective (Nikon CFI apochromat $\times 100,1.49$ numerical aperture). The collected fluorescence photons were passed through a blazed dispersive grating (100 grooves per mm, STAR100, Panton Kawskely Education Ltd.). The image was then split into a non-dispersed zero-order image and a spectrally dispersed first-order spectral image. Both the zero-order and the first-order images can be collected by an EMCCD camera (ProEM 532, Princeton Instruments) simultaneously. A calibration curve was obtained by fitting the wavelength information provided by a fluorescence calibration source with distinct emission peaks at 487.7, 546.5, 611.6, and $707.0 \mathrm{~nm}$ and the corresponding zeroth-order to first-order pixel location with a linear polynomial function. The spectral dispersion of the system was also determined to be $\sim 3 \mathrm{~nm} / \mathrm{pixel}$ from the slope of a calibration curve. The single-molecule emission spectra were calibrated using the single-molecule locations in the spatial channel as references and further interpolated to each wavelength unit from 500-800 nm. The EMCCD camera acquired images at an exposure time of $50 \mathrm{~ms}$.

The intensity-weighted spectral centroid $\left(\lambda_{\mathrm{sc}}\right)$ is calculated as the intensity-weighted average wavelength ${ }^{1}$ to minimize noise uncertainty contribution on the precision to calculate spectral features of every single-molecule spectra collecting with spectral widths of $150 \mathrm{~nm}$ using the equation below:

\section{Single-Molecule $\lambda_{\text {sc }}$ calculation}

For all the molecules except AF647, Rh110 and AO: $\lambda_{\text {sc }}=\sum_{n=525}^{n=675} I(n) W(n) / \sum_{n=525}^{n=675} W(n)$

For AF647: $\lambda \mathrm{sc}=\sum_{n=800}^{n=650} I(n) W(n) / \sum_{n=800}^{n=650} W(n)$

For AO and Rh110: $\lambda_{\mathrm{SC}}=\sum_{n=650}^{n=500} I(n) W(n) / \sum_{n=650}^{n=500} W(n)$

where $I(n)$ presents the intensity value at each wavelength after interpolation and $W(n)$ represents the wavelength.

\section{Calculating the smFSH contributions of different origins}

In probability theory, calculation of sum (Z) of normally distributed random variables $\mathrm{X}$ and $\mathrm{Y}$ follows

$\operatorname{Var}(\mathrm{Z})=\operatorname{Var}(\mathrm{X})+\operatorname{Var}(\mathrm{Y})$ 
where $\operatorname{Var}(\mathrm{X})$, $\operatorname{Var}(\mathrm{Y})$, and $\operatorname{Var}(\mathrm{Z})$ are the variances of $\mathrm{X}$, $\mathrm{Y}$ or $\mathrm{Z}$ respectively; the variance is the square of standard deviation.

In our study, the overall smFSH of Rhodamine B is the sum of multiple independent variables including the system's noise uncertainty, photoinduced spectral changes, conformation-induced spectral changes, etc. The model compound Rh101 provides negative controls that exclude the photoinduced and rotation-induced spectral variations. Thus, the smFSH measurements of Rh101 allow us to quantify the contributions of these origins. Specifically, the percentage contribution of rotamer (in variance) is calculated using the following equation according to probability theory:

$\operatorname{Var}($ rotamer contribution) $]=[\operatorname{Var}(\mathrm{RhB})-\operatorname{Var}(\mathrm{Rh} 101)] / \operatorname{Var}(\mathrm{RhB})$ 


\section{Supplementary Note 1 . The spectral precision calculation for the sSMLM system.}

We performed numerical simulation matching the exact setting of camera configuration and background in actual experiment acquisitions to calculate the precision (standard deviation value of $\lambda_{S C}$ ) to measure the intensity-weighted spectral centroid values using an established protocol ${ }^{2}$.

For the wide-slit configuration, the background is relatively high (total background $=800-1500$ photons, the gray bar in Figure S2A). Under this condition, the minimal spectral precision is $4.5 \mathrm{~nm}$ with a PCT of 500 for experimentally detected single molecules. Among different experimentally measured samples, the range of spectral precision we achieved is between 1.8-4.5 $\mathrm{nm}$ as indicated in the gray region in Figure S2B. For the narrow-slit configuration, the total background is relatively low (total background $=100-300$ photons, the yellow bar in Figure S2A) to achieve the minimal spectral precision of $2.5 \mathrm{~nm}$ and average spectral precision of 1-2 nm for all the dyes (the yellow highlighted region Figure S2B). The large smFSHs with STD >> $3 \mathrm{~nm}$ for the rhodamine dyes are not the noise uncertainty from the system.
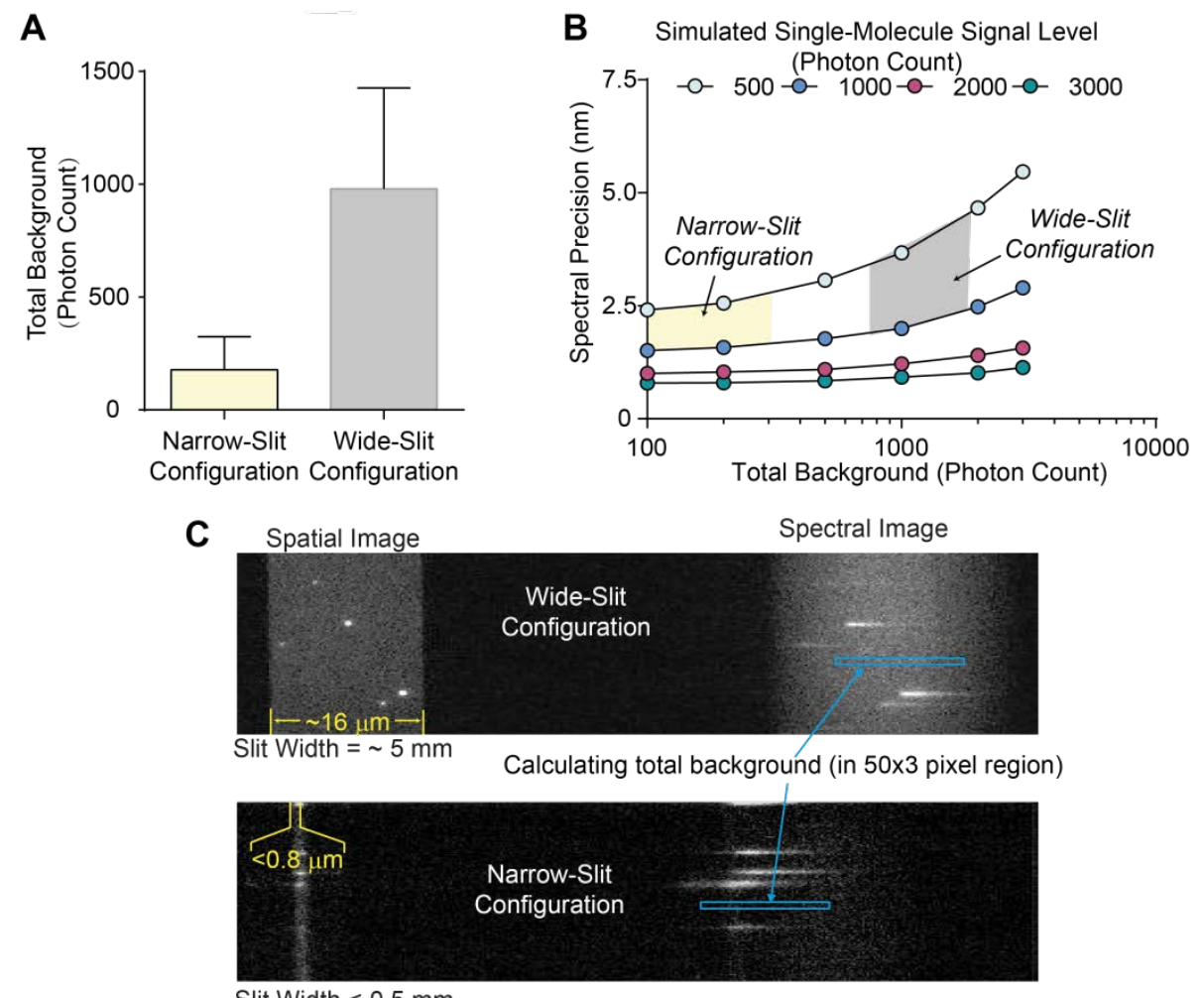

Figure S1. Comparisons of the total background level and spectral precision between the wide-slit and narrow-slit configurations. (A) The total background (from a $50 \times 3$ pixel region) of each configuration; (B) the calculated spectral precision as a function of the single-molecule signal level and the total background level; The yellow and gray areas are the ranges of spectral precisions achieved in the narrow-slit and wideslit configurations, respectively; (C) Representative frames in the wide-slit (top panel) and narrow-slit (bottom) configurations; the blue rectangular regions indicate the representative area ( $50 \times 3$ pixel) used to measure the total background level. 


\section{Supplementary Note 2. Minimally required spatial separation and achievable point density of single molecules}

\section{in the wide-slit and narrow-slit configurations.}

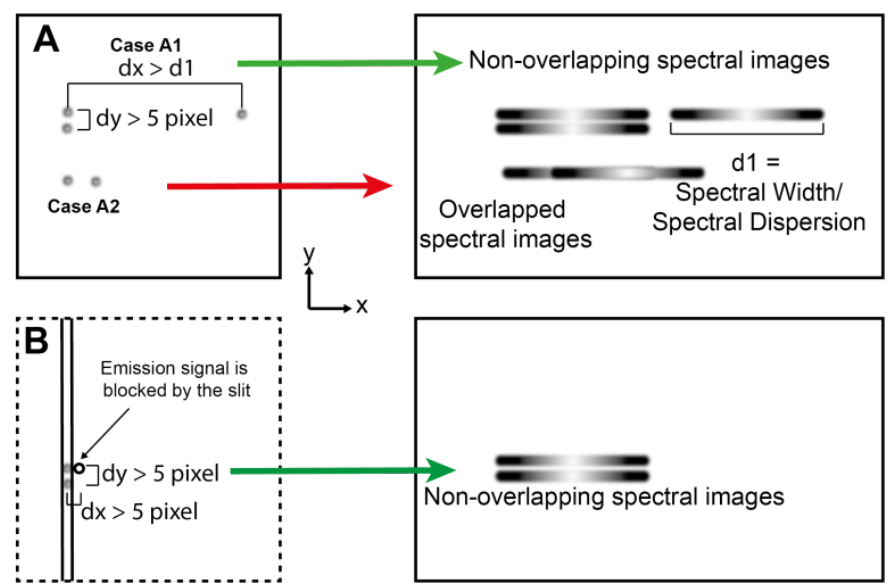

Figure S2. Schematic illustrations of the minimal spatial separation of the single molecules to achieve nonoverlapping spectral images in the wide-slit (A) and narrow-slit (B) configurations; (A) The X-coordinates difference (dx) and y-coordinates difference (dy) of two single molecules have to be greater than the spectral image width of the individual molecules (d1) and 5 pixels in our system to ensure non-overlapping spectra in the wide-slit configuration (Case $\mathrm{A} 1$ ); $\mathrm{dx}<\mathrm{d} 1$ results in overlapped single-molecule spectral images (Case A2); (B) In the narrow-slit configuration, dx only needs to be larger than 5 pixels to avoid overlapped spectral image as a result of confined filed-of-view in the spatial images using the narrow slit width; the emission signal of a molecule (arrow-pointed circle) is physically blocked by the slit even if the $\mathrm{dx}$ between this molecule and its adjacent one in the left is $<\mathrm{d} 1$.

In the existing sSMLM configuration with an entrance-slit width $>5 \mathrm{~mm}$ (referred to as the wide-slit configuration), single molecules are required to be rather separated to capture their non-overlapping spectral images to extract independent single-molecule emission spectra. We showed two representative cases in Figure S1A to calculate the minimal spatial separation and achievable point density of the single-molecule samples. As illustrated in Case A1, $\mathrm{x}$ coordinate difference $(\mathrm{dx})$ between two molecules at similar horizontal locations is required to be separated by the spectral image width (d1) so that their spectral images are not overlapped; d1 is equal to the emission spectral width of the dye molecule $(\sim 150 \mathrm{~nm})$ divided by the system's spectral dispersion $(3 \mathrm{~nm} /$ pixel). The y-coordinate difference (dy) between two molecules at similar vertical locations is required to be separated by 5 pixels (or $800 \mathrm{~nm}$, system's pixel size $=160 \mathrm{~nm}$ ), which is then determined by the point-spread-function width of our imaging system experimentally. Case A2 illustrates the condition that two molecules have overlapped emission spectra as a result of $\mathrm{dx}<=\mathrm{d} 1$. Considering that $\mathrm{dx}>>\mathrm{dy}$ and the single molecules are deposited on the substrate isotopically, $\mathrm{dx}$ determines the minimal spatial separation between single molecules. Consequently, the maximal point density that can be achieved in the wide-slit configuration is $1 / \mathrm{dx} 2=0.016 \mathrm{um}^{-2}$ or $64 \mathrm{um}^{2}$ per single molecule.

In the configuration designed in this study with an entrance-slit width $<50 \mathrm{~mm}$ (referred to as the narrow-slit configuration), the single molecules can still be relatively close to each other with non-overlapping spectral images. In Case B1 in Figure S1B, two molecules that have similar vertical locations can be unambiguously resolved in their spectral images if the dy $>=5$ pixels. The emission signal of a molecule (arrow-pointed circle) is physically blocked by the narrow slit width even if the $\mathrm{dx}$ between this molecule and its adjacent one in the left is relatively small ( 5 pixels $=<\mathrm{dx}<<\mathrm{d} 1)$. Therefore, the maximal point density in this condition is $1 /(\mathrm{dx} * \mathrm{dy})=1.6 \mathrm{um}^{-2}$ or $0.64 \mathrm{um}^{2}$ per single molecule. This maximal point density in the narrow-slit configuration is, therefore, 100 -fold higher than the one that can be achieved using the wide-slit configurations under our experimental condition, which significantly reduces the sample scanning time. 

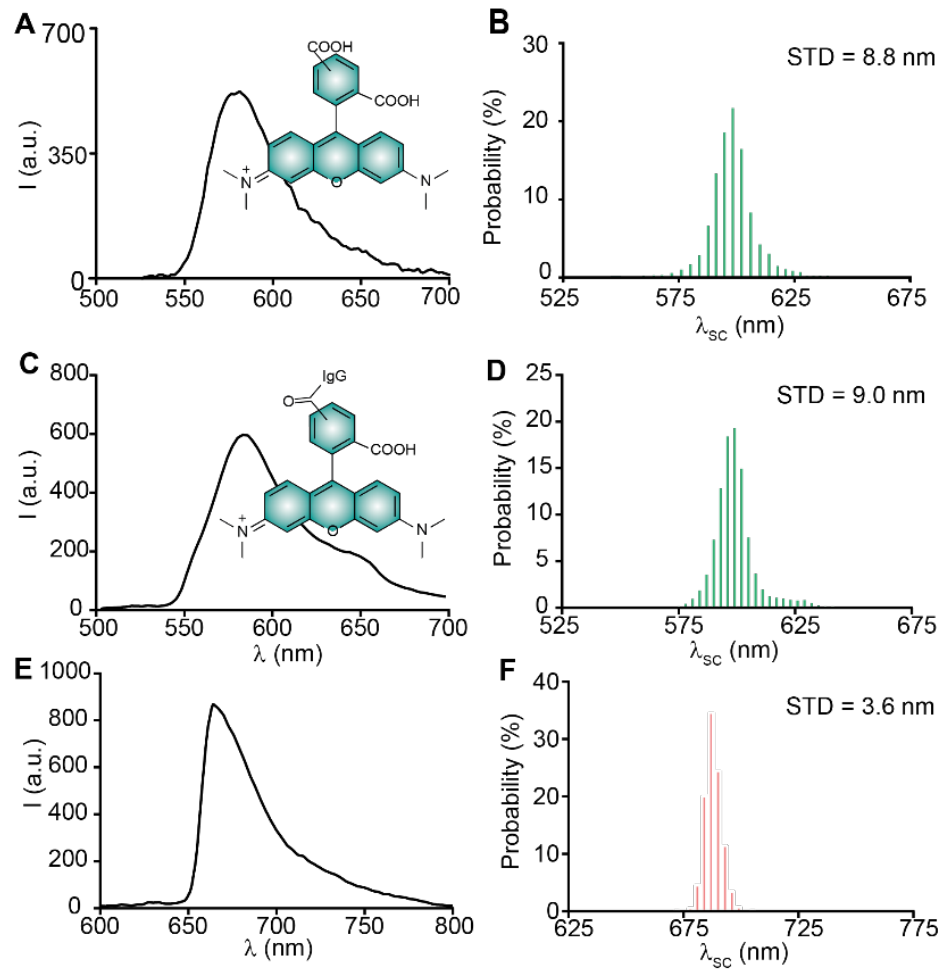

Figure S3. Characterizations smFSH properties of TAMARA-Glass, TAMRA-IgG, and AF647-Glass deposited on glass. (A-B) Averaged single-molecule emission spectrum (A) and histogram of probability distributions (B) of TAMRA directly deposited on glass substrates;(C-D) Averaged single-molecule emission spectrum (C) and histogram of probability distributions (D) of TAMRA molecule covalently conjugated to IgG protein and deposited on glass substrates; (E-F) Averaged single-molecule emission spectrum (E) and histogram of probability distributions (F) of AF647 directly deposited on glass substrates.

A Single-molecule spectral diffusion

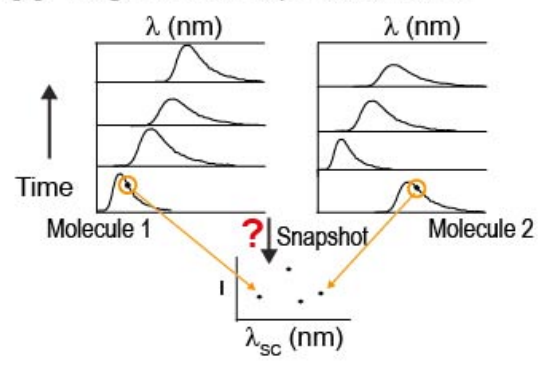

B Environment polarity change

C Conformational isomers
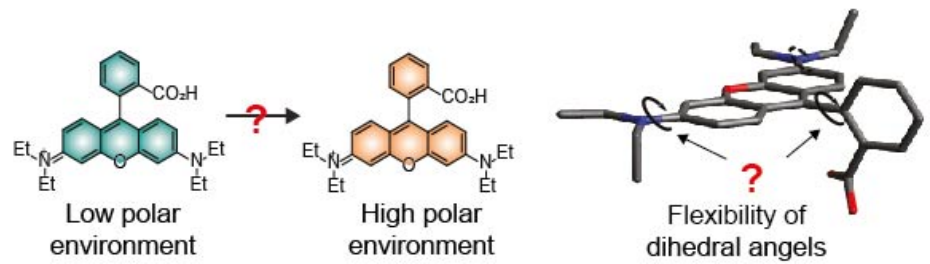

Figure S4. Hypothesized spectral variations to cause large smFSH in Rhodamine dyes from (A) the snapshots in different single-molecule spectral diffusion processes while all molecules demonstrate the large spectral variations comparable with smFSH; (B) environmental polarity changes to induce spectral red-shift from low polar to high polar environments; (C) conformational isomers caused by the flexibility of dihedral angles as indicated by the curved arrows. 

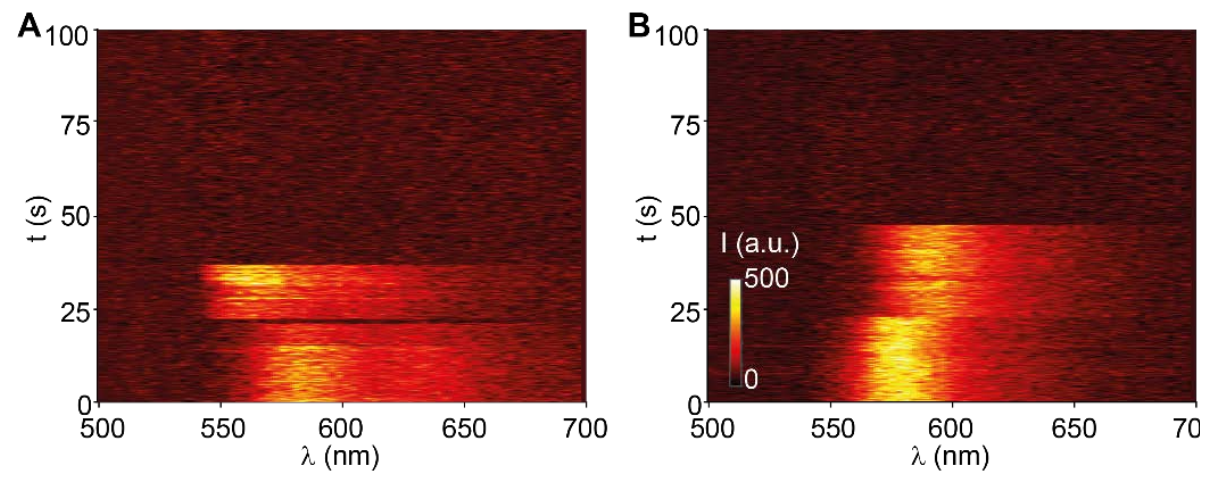

Figure S5. Representative single-molecule spectral diffusion events of RhB showing the significant hypochromic (A) and bathochromic (B) shifts in the spectral diffusion process.

A

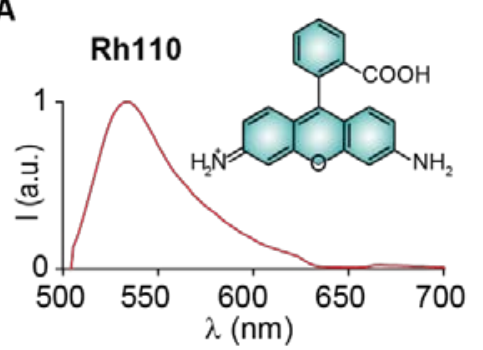

B

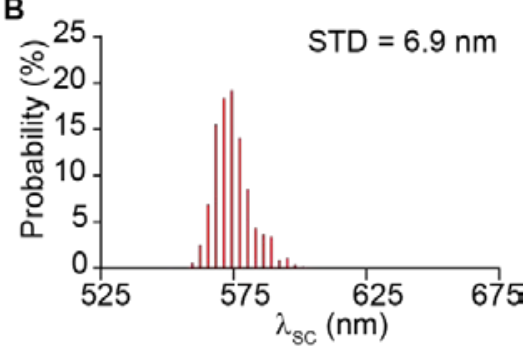

Figure S6. Characterizations smFSH properties of Rh110. Averaged single-molecule emission spectra (A) and histogram of probability distributions (B) of Rh110 deposited on glass substrates.

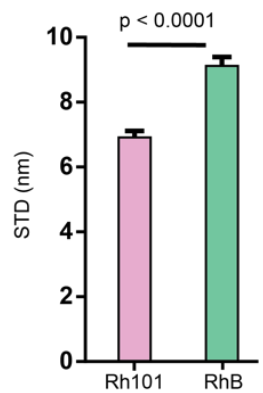

Figure S7. Statistical T-Test of STD between Rh101 and RhB measured on the glass surface. 


\section{Supplementary Note 3. Thermochemistry consideration.}

To understand the energy landscape and probability of conformers, we first calculated the ground state energy $(\Delta \mathrm{E})$ of the conformational isomers with a scan of the dihedral angles $\theta_{1}$ and $\theta_{2}$ in solution (Figure S8A). Notably, the bond lengths of $\mathrm{C}-\mathrm{N}$ bonds flanking the $\mathrm{N}, \mathrm{N}$-Diethylamino groups, and the Xanthene rings are 1.353 Angstrom and 1.355 Angstrom respectively, extracted from the optimized geometry. The literature-reported crystal structures of RhB showed consistent bond lengths of 1.356 Angstrom and 1.357 Angstrom for the two C-N bonds, respectively. ${ }^{3}$ These results indicate that both $\theta_{1}$ and $\theta_{2}$ have partial double bond characteristics with two-fold rotational symmetry. The optimized geometry and the conformation with $\theta_{1}$ or $\theta_{2}$ flipping $180^{\circ}$ have no more than $0.07 \mathrm{kcal}_{\text {mol}}{ }^{-1}$ difference in their $\Delta \mathrm{E}$. The perpendicular and planar conformations have at most $9.0 \mathrm{kcal} \mathrm{mol}^{-1}$ difference in their $\Delta \mathrm{E}((\mathrm{Figure} \mathbf{S 8 B}$ \& $\mathbf{C}$ and Table S1). We further computed the relative free Gibbs energy $(\Delta \mathrm{G})$ between the optimized and the transition
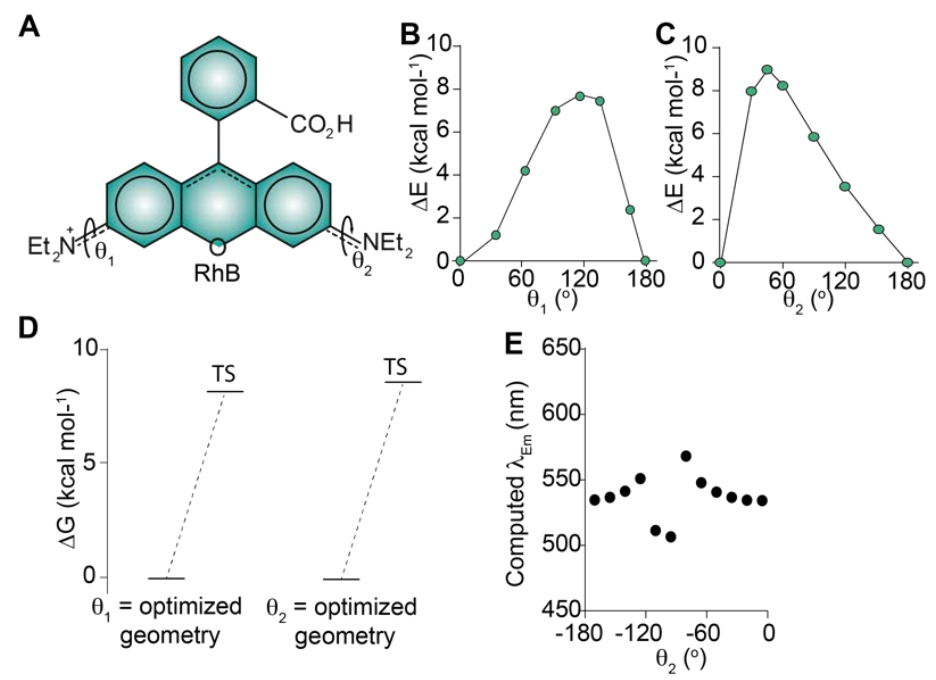

Figure S8. (A) Resonance structure of $\mathrm{RhB}$ and the free rotation of dihedral angles $\theta_{1}$ and $\theta_{2}$; (B-C) Computed ground state energy $(\Delta \mathrm{E})$ of $\mathrm{RhB}$ with the scan of $\theta_{1}(\mathbf{B})$ and $\theta_{2}(\mathbf{C})$ with an interval of $30^{\circ}$, respectively; (D) Computed free Gibbs energy $(\Delta \mathrm{G})$ of the conformational interconversions between the optimized geometry and the transition states state for flipping $\theta_{1}$ and $\theta_{2}$ by $180^{\circ}$; (D) Computed vertical excitation energy $\left(\lambda_{\mathrm{Em}}\right)$ value with the scan of dihedral angle $\theta_{2}$ with an interval of $15^{\circ}$.

state (TS) as the free Gibbs energy of activation (Figure S8D and Table S2). These computed activation energies for rotating $\theta_{1}$ or $\theta_{2}$ are 7.26 and $8.69 \mathrm{kcal} \mathrm{mol}^{-1}$, corresponding to rate constants $\mathrm{k}$ of $2.9 \times 10^{7} \mathrm{~s}^{-1}$ and $2.6 \times 10^{6} \mathrm{~s}^{-1}$ at 298 $\mathrm{K}$, respectively. Karstens et al reported consistently low activation energy (6.6 kcal mol-1) for rotating the N, N-Diethyl amino groups. ${ }^{4}$ All these results support that the $\mathrm{N}, \mathrm{N}$-Dialkylated amino groups in RhB can rotate rapidly (faster than once every millisecond) in solution at room temperature.

We believe that such free rotations in solution will populate a broad distribution of the dihedral angles on the glass surface and result in the 2.5-nm increase in STD value of RhB compared with Rh101. The computed $\lambda_{\text {Em }}$ with respect to varying $\theta_{2}$ shows such spectral variations (Figure S8D). From our smFSH measurement, we can detect rare conformers with probability as low as $0.01 \%$ (e.g., $\lambda_{\mathrm{sc}}=560$ and $630 \mathrm{~nm}$, or 35-nm away from $\lambda$ sc peak for RhB). We then calculated the energy $\left(\Delta \mathrm{E}_{\exp }\right)$ required to populate these conformers from the experimental result, which is $4.5 \mathrm{kcal} \mathrm{mol}^{-1}$ (corresponding to 7.5 times of $\mathrm{kT}$ at room temperature) according to the Boltzmann distribution $\mathrm{p} 35 / \mathrm{p}_{0}$ $=\mathrm{e}^{\Delta \mathrm{Eexp} / \mathrm{kT}}$, where $\mathrm{p}_{35}$ and $\mathrm{p} 0$ are the probabilities in the $\lambda_{\mathrm{sC}}=560 \& 630 \mathrm{~nm}$ and $\lambda_{\mathrm{sC}}=595 \mathrm{~nm}$, respectively, $\left(\mathrm{p}_{0}=\right.$ $18.39 \%)$. Using this energy as the higher bound for the computed $\Delta \mathrm{E}$, we believe that the dihedral angles can be anywhere from approximately $-180^{\circ}$ to $-150^{\circ}$ and $-75^{\circ}$ to $0^{\circ}$. The populated conformers with these dihedral angles result in a broad distribution of $\lambda_{\text {Em }}$ estimated from about 530-550 nm. After the adsorption to the glass surface, presumably, the interconversion of the rotamers significantly slows down or does not occur anymore upon RhB's interaction with the rigid glass surface. Otherwise, we will not observe a difference between the STD values of RhB and Rh101 using our 50 ms camera integration time; instead, we will observe a stable and broadened RhB emission spectrum because of the rapid interconversion (rotamers' half-life $<1 \mathrm{~ms}$ ) among different camera frames.

Notably, these computed thermochemistry parameters are calculated in free solution, while the detailed interactions and associated energy changes between the glass substrate and the Rhodamine molecules are neglected. 
It is challenging to simulate such energy changes with the existing glass model to obtain simulated $\lambda$ Em probability distribution, again, based on a Boltzmann distribution.

After the adsorption to the glass surface, presumably, the interconversion of the rotamers significantly slows down or does not occur anymore upon RhB's interaction with the rigid glass surface. Otherwise, we will not observe a difference between the STD values of RhB and Rh101 using our 50 ms camera integration time; instead, we will observe a stable and broadened RhB emission spectrum because of the rapid interconversion (because rotamers' halflife $<1 \mathrm{~ms}$ ) among different camera frames. 


\section{Computational Data}

Computational method. Density functional theory (DFT) calculations were carried out using Q-Chem ${ }^{5}$ version number 4.2.0. The M06 correlation functional was applied for geometry optimizations and vibrational frequency calculations with the $6-31 \mathrm{G}^{*}$ basis set ${ }^{6}$. The van der Waals correction was taken into account using Grimme's empirical dispersion potential. ${ }^{7}$ We first calculated the ground state electronic energy $(\Delta \mathrm{E})$ of conformations with an angular $\left(\theta_{2}\right)$ interval of $30^{\circ}$. Then we computed the free Gibbs energy $(\Delta \mathrm{G})$ of activation for the interconversion from optimized geometry to the geometry that $\theta_{2}$ flips $180^{\circ}$ through a transition state (TS) optimization and frequency calculation. Lastly, the computed $\lambda_{\mathrm{Em}}$ was calculated by the excitation energy on the geometry optimized on the potential energy surface of the first excited state by using linear response time-dependent density functional theory (TDDFT). A wavelength offset of $100 \mathrm{~nm}$ is added to all calculated $\lambda_{\mathrm{Em}}$ to match with experimental observation. In the calculation of $\lambda_{\mathrm{Em}}$ for the different dihedral angles in Figure S8A, conformations with an angular interval of $15^{\circ}$ are used along with the optimized geometry listed below. The solvent effect is taken into account by using the COSMO model. ${ }^{8}$ The dielectric constant of the solvent is 24.3 .

\section{Coordinates of the optimized geometry of Rhodamine B}

\begin{tabular}{|c|c|c|c|}
\hline $\mathrm{C}$ & 0.0287087434 & 4.4849184148 & 2.1271138132 \\
\hline $\mathrm{C}$ & 0.0185021831 & 3.1190130254 & 1.8863441767 \\
\hline $\mathrm{C}$ & 0.0166119801 & 2.6142701626 & 0.5916948413 \\
\hline $\mathrm{C}$ & 0.0525446816 & 3.5174455957 & -0.4800314231 \\
\hline $\mathrm{C}$ & 0.0634822201 & 4.8849308331 & -0.2287522418 \\
\hline $\mathrm{C}$ & 0.0438579404 & 5.3718736928 & 1.0673569493 \\
\hline $\mathrm{C}$ & 0.0018048502 & 1.1299782759 & 0.4068260600 \\
\hline $\mathrm{C}$ & -1.2099251312 & 0.4187723465 & 0.2633239845 \\
\hline $\mathrm{C}$ & -1.1940200603 & -0.9959409490 & 0.1201899054 \\
\hline $\mathrm{O}$ & -0.0265528186 & -1.6805462832 & 0.1412902197 \\
\hline $\mathrm{C}$ & 1.1543166410 & -1.0338356796 & 0.2509625761 \\
\hline $\mathrm{C}$ & 1.1973917858 & 0.3841533696 & 0.3944172759 \\
\hline $\mathrm{C}$ & 2.2681086209 & -1.8124097663 & 0.2178966427 \\
\hline $\mathrm{C}$ & 3.5687306912 & -1.2445793892 & 0.3344687380 \\
\hline $\mathrm{C}$ & 3.6298581866 & 0.1815148118 & 0.4696231257 \\
\hline $\mathrm{C}$ & 2.5056744494 & 0.9407637913 & 0.4998125915 \\
\hline $\mathrm{C}$ & -2.5021233353 & 1.0188713653 & 0.2381324795 \\
\hline $\mathrm{C}$ & -3.6394126193 & 0.2974295527 & 0.0867324949 \\
\hline $\mathrm{C}$ & -3.6069455129 & -1.1274651425 & -0.0750809425 \\
\hline $\mathrm{C}$ & -2.3200013220 & -1.7406404046 & -0.0340718514 \\
\hline $\mathrm{N}$ & 4.6728827443 & -2.0119206448 & 0.3216769219 \\
\hline $\mathrm{N}$ & -4.7212215718 & -1.8537424581 & -0.2587291250 \\
\hline $\mathrm{C}$ & -6.0457366706 & -1.2274152296 & -0.2023352965 \\
\hline $\mathrm{C}$ & -6.5692058768 & -1.0454910742 & 1.2225210535 \\
\hline $\mathrm{C}$ & -4.6074625019 & -3.3200083377 & -0.3664830155 \\
\hline $\mathrm{C}$ & -5.8876021223 & -4.0697867883 & -0.7132483750 \\
\hline $\mathrm{C}$ & 6.0086341298 & -1.4085056812 & 0.3184143475 \\
\hline $\mathrm{C}$ & 4.5267028078 & -3.4644743467 & 0.1176119323 \\
\hline $\mathrm{C}$ & 6.4650413213 & -0.9520716831 & -1.0674908989 \\
\hline $\mathrm{C}$ & 5.7975710072 & -4.2927666506 & 0.2594111714 \\
\hline $\mathrm{C}$ & 0.0287906926 & 3.0277437573 & -1.9018730634 \\
\hline $\mathrm{O}$ & -0.5974002897 & 2.0879341536 & -2.2547858453 \\
\hline $\mathrm{O}$ & 0.7515116364 & 3.7235749649 & -2.7744842093 \\
\hline $\mathrm{H}$ & 0.0201810866 & 4.8485090444 & 3.1382010578 \\
\hline $\mathrm{H}$ & 0.0067884612 & 2.4339546563 & 2.7146021110 \\
\hline $\mathrm{H}$ & 0.0514956401 & 5.5815168600 & -1.0482573339 \\
\hline $\mathrm{H}$ & 0.0373210228 & 6.4316044505 & 1.2425999998 \\
\hline $\mathrm{H}$ & 2.1199422283 & -2.8631251498 & 0.0929650102 \\
\hline $\mathrm{H}$ & 4.5731967270 & 0.6788042269 & 0.5441621914 \\
\hline $\mathrm{H}$ & 2.6025316604 & 2.0038560819 & 0.6063470394 \\
\hline $\mathrm{H}$ & -2.5722047838 & 2.0841204594 & 0.3379101442 \\
\hline $\mathrm{H}$ & -4.5705715050 & 0.8221707871 & 0.0831222304 \\
\hline $\mathrm{H}$ & -2.1947340802 & -2.7977471994 & -0.1253412642 \\
\hline
\end{tabular}




$\begin{array}{lrrr}\mathrm{H} & -6.0072222576 & -0.2831719462 & -0.7241987169 \\ \mathrm{H} & -6.7264006832 & -1.8404996392 & -0.7665075713 \\ \mathrm{H} & -7.5489808677 & -0.5802203972 & 1.1967361320 \\ \mathrm{H} & -6.6639862460 & -1.9999435906 & 1.7282446531 \\ \mathrm{H} & -5.9114810389 & -0.4182897691 & 1.8131595684 \\ \mathrm{H} & -3.8790350156 & -3.5392789401 & -1.1366905806 \\ \mathrm{H} & -4.2161816394 & -3.7066566794 & 0.5701333336 \\ \mathrm{H} & -5.6407340445 & -5.1241669635 & -0.7660123196 \\ \mathrm{H} & -6.6612547797 & -3.9551741918 & 0.0355446117 \\ \mathrm{H} & -6.2832538081 & -3.7795874155 & -1.6789843968 \\ \mathrm{H} & 6.0227741461 & -0.5877318754 & 1.0198209779 \\ \mathrm{H} & 6.7003720773 & -2.1330619344 & 0.7111637498 \\ \mathrm{H} & 3.8144311808 & -3.8300281032 & 0.8462019728 \\ \mathrm{H} & 4.1009688175 & -3.6370507907 & -0.8668312685 \\ \mathrm{H} & 7.4569010487 & -0.5170654140 & -1.0029555225 \\ \mathrm{H} & 6.5090908918 & -1.7867989792 & -1.7580912826 \\ \mathrm{H} & 5.7951214172 & -0.2091836458 & -1.4849020167 \\ \mathrm{H} & 5.5266430471 & -5.3311425206 & 0.1052164297 \\ \mathrm{H} & 6.5507464734 & -4.0420637143 & -0.4769146565 \\ \mathrm{H} & 6.2297152345 & -4.2122582202 & 1.2496072062 \\ \mathrm{H} & 1.3123364761 & 4.3639223192 & -2.3577696947\end{array}$

Table S1. Computed ground state energy $(\Delta \mathrm{E})$ of conformational isomers with the scan of $\theta_{2}$ or $\theta_{2}$ from $0^{\circ}$ to $180^{\circ}$ at an interval of $30^{\circ}$ while the other dihedral angle was fixed at optimized geometry.

\begin{tabular}{cc}
$\theta_{1}\left({ }^{\circ}\right)$ & $\Delta \mathrm{E}\left(\mathrm{kcal} \mathrm{mol}^{-1}\right)$ \\
\hline 0 & 0 \\
30 & 1.14 \\
60 & 4.22 \\
90 & 6.97 \\
120 & 7.33 \\
135 & 7.04 \\
165 & 2.63 \\
180 & 0.08 \\
\hline$\theta_{2}\left(^{\circ}\right)$ & $\Delta \mathrm{E}\left(\mathrm{kcal} \mathrm{mol}{ }^{-1}\right)$ \\
\hline 0 & 0 \\
30 & 7.98 \\
45 & 8.99 \\
60 & 8.23 \\
90 & 5.85 \\
120 & 3.54 \\
150 & 1.68 \\
180 & 0.002 \\
\hline
\end{tabular}

Table S2. Computed free Gibbs energy $(\Delta G)$ of the energy barrier for flipping $\theta_{1}$ or $\theta_{2} 180^{\circ}$.

\begin{tabular}{cc}
$\theta_{1}\left({ }^{\circ}\right)$ & $\Delta \mathrm{G}(\mathrm{kcal} \mathrm{mol}-1)$ \\
\hline $\mathrm{TS}$ & 7.26 \\
\hline$\theta_{2}\left({ }^{\circ}\right)$ & $\Delta \mathrm{G}\left(\mathrm{kcal} \mathrm{mol}^{-1}\right)$ \\
\hline $\mathrm{TS}$ & 8.69 \\
\hline
\end{tabular}


Table S3. Dihedral angle scan of the two N, N-Diethylamino groups of RhB

\begin{tabular}{ccc}
$\theta_{2}\left({ }^{\circ}\right)$ & $\lambda_{\mathrm{Em}}$ & $f$ \\
\hline-170.502 & 534.7424 & 0.88 \\
-155.502 & 536.7178 & 0.8732 \\
-140.502 & 541.3348 & 0.847 \\
-125.502 & 551.1633 & 0.7732 \\
-110.502 & 511.4429 & 0.4952 \\
-95.502 & 506.5455 & 0.3943 \\
-80.502 & 568.2183 & 0.5004 \\
-65.502 & 547.8389 & 0.7268 \\
-50.502 & 540.77 & 0.8148 \\
-35.502 & 536.764 & 0.8519 \\
-20.502 & 534.6662 & 0.8695 \\
-5.502 & 534.3312 & 0.8764 \\
\hline
\end{tabular}

\section{Supporting Reference}

1. Zhang, Y.; Song, K.-H.; Dong, B.; Davis, J. L.; Shao, G.; Sun, C.; Zhang, H. F. Multicolor super-resolution imaging using spectroscopic single-molecule localization microscopy with optimal spectral dispersion. Appl. Opt. 2019, 58, 2248-2255.

2. $\quad$ Song, K.-H.; Dong, B.; Sun, C.; Zhang, H. F. Theoretical analysis of spectral precision in spectroscopic single-molecule localization microscopy. Rev. Sci. Instrum. 2018, 89, 123703

3. $\quad$ Kvick, A.; Vaughan, G. B. M.; Wang, X.; Sun, Y.; Long, Y. A synchrotron-radiation study of the lactone form of rhodamine B at 120K. Acta Cryst. 2000, C56, 1232-1233

4. Karstens, T.; Kobs, K. Rhodamine B and rhodamine 101 as reference substances for fluorescence quantum yield measurements. J. Phys. Chem. 1980, 84, 1871-1872.

5. Shao, Y.; et al. Advances in molecular quantum chemistry contained in the Q-Chem 4 program package. Mol. Phys. 2014, 113, 184-215

6. Zhao, Y.; Truhlar, D. G. The M06 suite of density functionals for main group thermochemistry, thermochemical kinetics, noncovalent interactions, excited states, and transition elements: two new functionals and systematic testing of four M06-class functionals and 12 other functionals. Theor. Chem. Acc. 2008, 120, 215-241

7. Grimme, S. Semiempirical GGA - type density functional constructed with a long - range dispersion correction. J. Comput. Chem., 2006, 27, 1787-1799

8. Schuurmann, G., Klamt, A. COSMO: a new approach to dielectric screening in solvents with explicit expressions for the screening energy and its gradient. J. Chem. Soc., Perkin Trans. 2, 1993, 799-805 